NONCOMMUTATIVE GEOMETRY

AND QUANTUM GROUPS

BANACH CENTER PUBLICATIONS, VOLUME 61

INSTITUTE OF MATHEMATICS

POLISH ACADEMY OF SCIENCES

WARSZAWA 2003

\title{
HOPF COHOMOLOGY VANISHING VIA APPROXIMATION BY HOCHSCHILD COHOMOLOGY
}

\author{
AKIRA MASUOKA \\ Institute of Mathematics, University of Tsukuba, Ibaraki 305-8571, Japan \\ E-mail: akira@math.tsukuba.ac.jp
}

Introduction. The introductory part of the beautiful lectures given by H. -J. Schnei$\operatorname{der}^{1}$ at the conference reminded us of one of the original motivations of Hopf algebra theory, that is, to simplify and generalize (or quantize, in more recent language) the theory of affine group schemes, by studying Hopf algebras. One of the most successful results in Hopf algebra theory can be found in the study of cleft comodule algebras, or in other words Hopf-crossed products. In this paper we give a new approach from the cleftness results to the theorems by Nagata, by Takeuchi [T1], and by Sullivan [Su], which all are directly connected to the theory of affine group schemes; see [A, Chap. 4].

For a Hopf algebra $H$, a right $H$-comodule algebra $A$ is said to be cleft [Sw1] if there is a convolution-invertible $H$-colinear map $H \rightarrow A$. Such an algebra $A$ is characterized as a Hopf-Galois extension with normal basis, and also as a Hopf-crossed product [DT; BCM]; the crossed product is given by a measuring action and a non-abelian Hopf 2-cocycle. Therefore, cleft comodule algebras are studied, regarded as an important, special class of Hopf-Galois extensions [DT; BM], as a generalized object unifying various constructions of rings such as group-crossed products or Ore extensions [BCM], and as an interesting example of non-abelian cohomology [D3]. A sequence $K \rightarrow A \rightarrow H$ of Hopf algebras is called a cleft extension [S1], if there is a left $K$-linear and right $H$-colinear isomorphism $K \otimes H \cong A$. Then $A$ is not only cleft as a right $H$-comodule algebra, but also cocleft, in the dual situation, as a left $K$-module coalgebra, and hence is described as a bicrossed product of $K$ and $H$; see Section 1 below. Such extensions are studied in the abelian situation from the view-point of abelian cohomology [S1; $\mathrm{Si} ; \mathrm{H} ; \mathrm{M} 2]$, and also in the non-abelian situation toward applications to quantum groups [Mj1; $\mathrm{AD}]$.

2000 Mathematics Subject Classification: Primary 16W30.

The paper is in final form and no version of it will be published elsewhere.

${ }^{1}$ He started with an old result by Grothendieck on torsors for finite affine group schemes, and then explained how it is generalized in the non-commutative context by the Kreimer-Takeuchi Theorem [KT, Thm. 1.7], with a proof much simplified. 
Difficulties of Hopf-crossed products arise from the fact that the relevant Hopf cohomology is non-abelian and multiplicative. However, as we will see in the proof of Theorem 4.1, the Hopf cohomology can be approximated, in some sense, by the Hochschild cohomology, which is abelian and additive. By this idea we prove in Theorem 3.1 that if the coradical $K$ of a Hopf algebra $A$ forms a Hopf subalgebra, the left $K$-module coalgebra $A$ is isomorphic to a cosmash product $K \ll A / K^{+} A$, that is, a crossed coproduct with trivial dual cocycle; this was proved by Stefan and Van Oystaeyen [SV] when ch $k=0$ and $K$ is finite-dimensional, along the same line of the proof of the Wedderburn-Malcev Theorem. Theorem 3.1 is the starting point of our new approach, given in Section 3, to Takeuchi's and Sullivan's Theorems. The same idea will be used in Section 5 to prove vanishing of the cohomology associated to an abelian matched pair [Si; T3] (or a Singer pair) of Hopf algebras.

Conventions. We work over a fixed field $k$. Let $C$ be a coalgebra. Its structure maps are denoted by $\Delta\left(=\Delta_{C}\right)$, $\varepsilon$, as usual. We let $C^{+}$denote the kernel Ker $\varepsilon$ of the counit. We use the Sweedler notation of the form $\Delta(c)=c_{1} \otimes c_{2}$. For an algebra $R$, the convolution product of linear maps $C \rightarrow R$ is denoted by $*$. The convolution-inverse of $\phi$ is denoted by $\phi^{-}$, if it exists.

1. Preliminaries - Cleftness results. Fix a Hopf algebra $H$ and an algebra $R$. Let $A$ be a right $H$-comodule algebra whose subalgebra $A^{\text {co } H}$ of $H$-coinvariants equals $R$. $A$ is said to be cleft if there exists an $H$-colinear map $\phi: H \rightarrow A$ which is invertible under the convolution product. We will suppose $\phi$ preserves the unit, that is, $\phi(1)=1$; this is possible by replacing $\phi$ with $\phi^{-}(1) \phi$. We have then a left $R$-linear and right $H$-colinear isomorphism [DT, Thm. 9]

$$
\tilde{\phi}: R \otimes H \stackrel{\cong}{\longrightarrow} A, \quad \tilde{\phi}(x \otimes a)=x \phi(a)
$$

which preserves the unit.

Define

$$
\begin{array}{ll}
\rightarrow: H \otimes R \rightarrow R, & a \rightarrow x=\phi\left(a_{1}\right) x \phi^{-}\left(a_{2}\right), \\
\sigma: H \otimes H \rightarrow R, & \sigma(a, b)=\phi\left(a_{1}\right) \phi\left(b_{1}\right) \phi^{-}\left(a_{2} b_{2}\right),
\end{array}
$$

which then form a system of crossed product [D3]. This means that $\rightarrow$ is a measuring action, $\sigma$ is an invertible linear map, and these satisfy the twisted module condition and the cocycle condition given by (2), (1) in [DT, Lemma 10] (see also [BCM]) as well as the normalization condition

$$
1 \rightarrow x=x, \quad \sigma(a, 1)=\varepsilon(a) 1=\sigma(1, a),
$$

where $x \in R, a \in H$. These conditions are equivalent to that the right $H$-comodule $R \otimes H$ forms a right $H$-comodule algebra with unit $1 \otimes 1$ with respect to the product

$$
(x \otimes a)(y \otimes b)=x\left(a_{1} \rightarrow y\right) \sigma\left(a_{2}, b_{1}\right) \otimes a_{3} b_{2},
$$

where $x \otimes a, y \otimes b \in R \otimes H$. This is called a crossed product, and denoted by $R \rtimes_{\sigma} H$ in this paper, following Majid [Mj2]. The isomorphism $\tilde{\phi}: R \rtimes_{\sigma} H \stackrel{\cong}{\longrightarrow} A$ is now one of $H$-comodule algebras. See [DT; BCM], and also [Mo, Chap. 7; P]. 
The assignment $(A, \phi) \mapsto(\rightarrow, \sigma)$ given above provides a bijection from the set of the isomorphism classes of all pairs $(A, \phi)$ to the set of all systems $(\rightarrow, \sigma)$ of crossed product.

The definitions and the results are formally dualized [MD; DMR]. A left $H$-module coalgebra $C$ is said to be cocleft, if there exists an invertible $H$-linear map $\gamma: C \rightarrow H$ which preserves the counit. Let $E=C / H^{+} C$. Such a map $\gamma$ corresponds to a system $(\rho, \tau)$ which makes $H \otimes E$ into a coalgebra, $H^{\tau} \ll E$, of crossed coproduct. In particular, $\rho: E \rightarrow E \otimes H, \rho(x)=x_{E} \otimes x_{H}$ is a comeasuring coaction in the sense that

$$
\Delta\left(x_{E}\right) \otimes x_{H}=x_{1 E} \otimes x_{2 E} \otimes x_{1 H} x_{2 H}, \quad \varepsilon\left(x_{E}\right) x_{H}=\varepsilon(x) 1,
$$

where $x \in E$. If $\gamma$ is a coalgebra map, or equivalently $\tau$ is trivial in the sense $\tau=\varepsilon$ $(=\varepsilon(\quad) 1 \otimes 1)$, then $H^{\tau}<E$ is a cosmash product [D1], which is denoted simply by $H \ltimes E$.

A sequence $K \rightarrow A \rightarrow H$ of Hopf algebras is called a cleft extension of $H$ by $K$ [S1] if there exists a left $K$-linear and right $H$-colinear isomorphism

$$
\alpha: K \otimes H \stackrel{\cong}{\longrightarrow} A,
$$

which can preserve the unit and counit. Then $\phi: H \rightarrow A, \phi(h)=\alpha(1 \otimes h)$ and $\gamma: A \rightarrow K$, $\gamma(a)=(1 \otimes \varepsilon) \circ \alpha^{-1}(a)$ are both invertible [MD, Prop. 3.2; M1, Prop. 3.10]. Hence there exist uniquely a system $(\rightarrow, \sigma)$ of crossed product and a system $(\rho, \tau)$ of crossed coproduct such that the 'bicrossed product'

$$
K^{\tau} \triangleleft_{\sigma} H,
$$

being $K \rtimes_{\sigma} H$ as an algebra, and $K^{\tau} \ll H$ as a coalgebra, forms a Hopf algebra, and $\alpha: K^{\tau} \triangleleft_{\sigma} H \stackrel{\cong}{\longrightarrow} A$ is a Hopf algebra isomorphism; see [Mj1; AD].

To form a bialgebra, the systems $(\rightarrow, \sigma)$ and $(\rho, \tau)$ should satisfy some compatibility conditions as given in [AD, Thm. 2.20]. If $H$ is cocommutative and $K$ is commutative, then $\rightarrow$ and $\rho$ are independent of the choice of $\alpha$, and they should satisfy such a condition that $(H, K, \rightarrow, \rho)$ forms an abelian matched pair [T3, Def. 1.1], or a Singer pair as we will call it in this paper. The equivalence classes of those cleft extensions which are associated as above to a fixed Singer pair $(H, K, \rightarrow, \rho)$ form an abelian $\operatorname{group}, \operatorname{Opext}(H, K, \rightarrow, \rho)$; this is isomorphic to some cohomology group $H^{2}(H, K, \rightarrow, \rho)$. See [Si; H; M2] and Section 5 below.

\section{An alternative proof of Nagata's Theorem using the cleftness results.} The next theorem is what we call here Nagata's Theorem, obeying some custom. In fact this generalizes his result $[\mathrm{N}]$ on affine algebraic groups to commutative Hopf algebras which do not necessarily correspond to such groups; this was proved by Demazure and Gabriel [DG] and by Sweedler [Sw3] (see also [A, Chap. 4, Sect. 6]), independently. Later, Chin [C] gave a new proof; see also [Mo, Sect. 5.7].

Theorem 2.1 [DG; Sw3]. Suppose the characteristic ch $k=p>0$. A commutative cosemisimple Hopf algebra $A$ is cocommutative if its separable part $\pi_{0}(A)=k$.

As in $[\mathrm{C} ; \mathrm{Mo}]$, we completely follow [Sw3] to reduce the proof to the case when $A$ is finite-dimensional. Let $A$ be as above. We may suppose $A$ is finitely generated. Then $\pi_{0}(A)=k$ is equivalent to that $A$ (or its prime spectrum $\operatorname{Spec} A$ ) is connected. It is proved 
in [Sw3] that, given a finite-dimensional subcoalgebra $C \subset A$, there is a finite-dimensional quotient Hopf algebra $H$ of $A$ such that the composite $C \hookrightarrow A \rightarrow H$ of the natural maps is an injection; $H$ is necessarily commutative, cosemisimple and connected. Therefore, the theorem follows if we apply the next proposition to the base extension $\bar{k} \otimes H^{*}$ of the dual Hopf algebra $H^{*}$ of $H$.

Proposition 2.2. Suppose $k$ is an algebraically closed field of characteristic $p>0$. A finite-dimensional cocommutative Hopf algebra $A$ is of the form $(k G)^{*}$ with an abelian p-group $G$, if it is semisimple and irreducible.

Proof. We prove this by induction on $\operatorname{dim} A$. We may suppose $\operatorname{dim} A>1$. Then the restricted Lie algebra $\mathfrak{g}=P(A)$ of all primitives in $A$ is non-zero since $A$ is irreducible. The restricted envelope $B:=u(\mathfrak{g})$ of $\mathfrak{g}$ is embedded in $A$, and forms a normal Hopf subalgebra $\supsetneqq k$; see [Mo, Lemma 5.7.2 (3)]. If $B=A$, the result follows from Hochschild's Theorem; see [C; Mo, Thm. 2.3.3] also for a nice proof due to Chin.

Suppose $B \varsubsetneqq A$. By the induction hypothesis, the quotient Hopf algebra $\bar{A}:=A / B^{+} A$ is of the form $(k G)^{*}$, where $G$ is a $p$-group. Since $G$ has a subgroup $\mathbb{Z}_{p}$ of order $p$, there is a quotient Hopf algebra $H$ of $\bar{A}$ (and hence of $A$ ) such that

$$
H=\left(k \mathbb{Z}_{p}\right)^{*} \cong k[x] /\left(x^{p}-x\right)
$$

in which $x$ is primitive.

Let $K$ denote the Hopf subalgebra in $A$ of $H$-coinvariants. By the induction hypothesis, $K=(k F)^{*}$, where $F$ is an abelian p-group. By [S2, Thm. 2.2] (or [MD, Thm. 3.5; Mo, Cor. 8.4.7]), a finite-dimensional Hopf algebra is cleft as a right comodule algebra for any quotient Hopf algebra. Hence we have $A=K \rtimes_{\sigma} H$, a crossed product. The associated action $\rightarrow: H \otimes K \rightarrow K$ must be trivial. In fact, its dual coaction (see (1.1))

$$
\lambda=(-)^{*}: k F \rightarrow H^{*} \otimes k F
$$

maps each $a \in F$ to a grouplike in the $H^{*}$-Hopf algebra $H^{*} \otimes k F$ (or, under a natural identification, to an algebra map $\left.(k F)^{*} \rightarrow H^{*}\right)$, and so $\lambda(a)=1 \otimes a$, since $H^{*}=k \mathbb{Z}_{p}$ is connected. We see thus $K$ is central in $A$. Write $X=1 \rtimes x$ in $A$. We may suppose $X^{i}=1 \rtimes x^{i}$ for $0 \leq i<p$, by re-choosing the map $\phi$. It follows that $X$ and $K$ generates $A$, so that $A$ is commutative. (In fact we see $K[X] /\left(X^{p}-X-c\right) \cong A$, where $c=X^{p}-X \in K$.) Being in addition semisimple, cocommutative and of $p$-power dimension, $A$ is of the desired form.

3. A new approach to Takeuchi's and Sullivan's Theorems. Our proofs, presented below, of the two theorems are not quite new, but follow in large part the original proofs, depending especially on Takeuchi's idea of reduction [T1, Prop. 4]. A new point is only to use the following.

TheOREm 3.1. Let $A$ be a Hopf algebra whose coradical, say $K$, forms a Hopf subalgebra. Then the left $K$-module coalgebra $A$ is cosmash in the sense that there exists a $K$-linear coalgebra map $\gamma: A \rightarrow K$, and hence $A \cong K \ll A / K^{+} A$, a cosmash product.

Here we can suppose $\gamma(1)=1$, so that $\gamma$ is a left $K$-linear coalgebra map such that the restriction $\left.\gamma\right|_{K}$ onto $K$ is the identity map. This theorem will be proved in the next 
section. Stefan and Van Oystaeyen [SV, Cor. 2.13] prove this when $\operatorname{ch} k=0$ and $K$ is finite-dimensional.

Theorem 3.2 (Takeuchi [T1]). Suppose ch $k=0$. Let $A$ be a commutative Hopf algebra with coradical $K$. Then $K$ is a Hopf subalgebra of $A$, and there is a Hopf algebra map $\pi: A \rightarrow K$ such that $\left.\pi\right|_{K}=\mathrm{id}$.

This generalizes the Chevalley decomposition for affine algebraic groups.

To prove Theorem 3.2, suppose ch $k=0$. It is known [DG, IV, Sect. 3, Cor. 3.5] about linear representations of an abstract group $G$ that, if two representations on $V_{i}(i=1,2)$ of finite dimension are completely reducible, so is the tensor product representation on $V_{1} \otimes V_{2}$. This implies that the coradical $K^{\prime}$ of the dual Hopf algebra $(k G)^{\circ}$ of $k G$ is a Hopf subalgebra. Let $A \supset K$ be as above. To see that $K$ is a Hopf subalgebra, we may suppose that $A$ is finitely generated, and also that $k$ is algebraically closed since $k$ is perfect so that $\operatorname{Corad}(\bar{k} \otimes A)=\bar{k} \otimes K$. Then $A$ is regarded as a Hopf subalgebra of $(k G)^{\circ}$, where $G=\operatorname{Alg}(A, k)$; see [Mo, 9.3.2]. Hence, $K\left(=K^{\prime} \cap A\right)$ is a Hopf subalgebra; cf. the proof of [T1, Prop. 0].

To prove the remaining part of the theorem, we suppose first that $k$ is arbitrary and $A$ is a commutative Hopf algebra whose coradical $K$ is a Hopf subalgebra. Introduce the natural order among the pairs $(B, \varpi)$, where $B \subset A$ is a Hopf subalgebra including $K$, and $\varpi: B \rightarrow K$ is a Hopf algebra map such that $\left.\varpi\right|_{K}=$ id. By Zorn's Lemma, there exists a maximal pair $(B, \varpi)$. Suppose $B \varsubsetneqq A$ to see a contradiction.

Let $H=A / B^{+} A$, a quotient Hopf algebra; this is irreducible since $K \subset B$. Since $A$ is $B$-faithfully flat, it follows by [T2, Thm. 3] that $A$ is an injective right $H$-comodule. So, the unit $k \rightarrow A$ can be extended to a right $H$-colinear map $H \rightarrow A$, which is necessarily invertible and can preserve the counit. Hence we have $A=B^{\tau} \triangleleft_{\sigma} H$, a bicrossed product. The associated action is trivial since $A$ is commutative. The associated coaction $\rho: H \rightarrow H \otimes B$ maps each primitive $x \in P(H)$ to a primitive in the $B$-Hopf algebra $H \otimes B$ (see (1.1)), and so $\rho(P(H)) \subset P(H) \otimes B$. Notice $P(H) \neq 0$ since $H \neq k$. By the compatibility condition $[\mathrm{AD},(2.22)]$ between $\sigma$ and $\rho$, we have

$$
\left(1 \otimes \sigma\left(a_{1}, b_{1}\right)\right) \rho\left(a_{2} b_{2}\right)=\rho\left(a_{1}\right) \rho\left(b_{1}\right)\left(1 \otimes \sigma\left(a_{2}, b_{2}\right)\right),
$$

where $a, b \in H$. Therefore, if $J \subset H$ is a cocommutative Hopf subalgebra, then $\left.\rho\right|_{J}: J \rightarrow$ $H \otimes B$ is an algebra map. Suppose in particular, $J$ is the cocommutative Hopf subalgebra generated by $P(H)$. Then $\rho(J) \subset J \otimes B$.

We see $B^{\prime}:=B^{\tau} \triangleleft_{\sigma} J$ is a Hopf subalgebra of $A$, where the associated cocycle and dual cocycle are induced from $\sigma, \tau$, being denoted still by the same symbols. Let $I=(\operatorname{Ker} \varpi)$ be the Hopf ideal in $B^{\prime}$ generated by $\operatorname{Ker} \varpi$. Then $B / \operatorname{Ker} \varpi=K$, and so

$$
B^{\prime} / I=K^{\bar{\tau}} \triangleleft_{\bar{\sigma}} J,
$$

where the associated coaction $\bar{\rho}$ as well as $\bar{\sigma}, \bar{\tau}$ are induced from $\rho, \sigma, \tau$, respectively. By Theorem 3.1, we may suppose $\bar{\tau}$ is trivial.

Suppose now $\operatorname{ch} k=0$. Then $J=S(P(H))$, the symmetric algebra of $P(H)$. Let $\left\{x_{\lambda}\right\}(\neq \emptyset)$ be a basis of $P(H)$. Since the algebra map $J \rightarrow K \triangleleft_{\bar{\sigma}} J=B^{\prime} / I$ determined by $x_{\lambda} \mapsto 1 \triangleleft x_{\lambda}$ is invertible and $J$-colinear, this induces a $K$-algebra isomorphism 
$K \otimes J \stackrel{\cong}{\longrightarrow} B^{\prime} / I$. This is obviously a Hopf algebra isomorphism (and so $\bar{\sigma}$ is trivial), if we regard $K \otimes J=K \diamond J$, the cosmash product given by $\bar{\rho}$. Hence we have a Hopf algebra map $B^{\prime} \rightarrow B^{\prime} / I \rightarrow K$ which is identical on $K$. This contradicts the maximality of $(B, \varpi)$, concluding the proof of Takeuchi's Theorem.

TheOREm 3.3 (Sullivan [Su]). Suppose $k$ is an algebraically closed field of positive characteristic. Let $A$ be a commutative pointed Hopf algebra with coradical $K$, so that $K$ is a grouplike Hopf subalgebra. Then there is a Hopf algebra map $\pi: A \rightarrow K$ such that $\left.\pi\right|_{K}=\mathrm{id}$.

In the preceding proof, suppose $K$ is grouplike. Then there exist a grouplike $g$ in $K$ and a primitive $x \neq 0$ in $H$ such that $\rho(x)=x \otimes g$. We can take $J=k[x](\subset H)$, and then obtain as $B^{\prime} / I$ the commutative pointed Hopf algebra $K \triangleleft_{\bar{\sigma}} k[x]$ of bicrossed product, in which the action and the dual cocycle are both trivial, and the coaction is given by $\bar{\rho}(x)=x \otimes g$. To prove Sullivan's Theorem, we may suppose $A=K \triangleleft_{\bar{\sigma}} k[x]$ from the beginning. If $x$ is transcendental (over $k$ ), we can do as in the preceding proof.

Suppose $\operatorname{ch} k=p>0$ and $x$ is algebraic. We see that the minimal polynomial $f(x)$ of $x$ in $J=k[x]$ is of the form

$$
f(x)=c_{0} x+c_{1} x^{p}+\cdots+x^{p^{m}},
$$

where $c_{i} \in k(0 \leq i<m)$; this is characterized by the relation $f(x)=0$ in $P(J)$ with $m$ minimal. Write $X=1 \triangleleft \triangleleft x$ in $A$. Then we have

$$
\Delta(X)=X \otimes g+1 \otimes X, \quad \varepsilon(X)=0 .
$$

Let $q=p^{m}$. Since $\bar{\rho}$ is an algebra map, we have

$$
c_{0} x \otimes g+c_{1} x^{p} \otimes g^{p}+\cdots+x^{q} \otimes g^{q}=\bar{\rho}(f(x))=0 .
$$

This implies that

$$
g^{p^{i}}=g^{q} \text { whenever } c_{i} \neq 0 .
$$

Hence, $f(X \otimes g)=f(X) \otimes g^{q}$, and $f(b g)=f(b) g^{q}$ for $b \in k$. The former implies

$$
\Delta(f(X))=f(X) \otimes g^{q}+1 \otimes f(X) .
$$

Since $f(X)$, being right $J$-coinvariant, is in $K$,

$$
f(X)=c\left(1-g^{q}\right)
$$

for some $c \in k$.

If $k$ is algebraically closed, there exists $b$ in $k$ such that $f(b)=c$, and so $f(X)=$ $f(b(1-g))$. We have a right $J$-colinear algebra map $J=k[x] \rightarrow A$ determined by $x \mapsto X-b(1-g)$. Similarly to the last part of the preceding proof, this induces a Hopf algebra isomorphism $K \prec k[x] \stackrel{\cong}{\longrightarrow} A$ which is identical on $K$, and so we have a desired $\operatorname{map} \pi: A \rightarrow K$.

4. Extension of cosmash products. For a Hopf algebra $K$, we let $\operatorname{Ext}_{K}^{n}(\quad, \quad)$ denote the $n$-th Ext group for the abelian category of right $K$-comodules. We regard $k$ as a trivial right $K$-comodule. 
TheOREM 4.1. Let $K$ be a Hopf algebra such that $\operatorname{Ext}_{K}^{2}(V, k)=0$ for any right $K$ comodule $V$. Let $C$ be a cocleft left $K$-module coalgebra. Let $D \subset C$ be a $K$-stable subcoalgebra including the coradical Corad $C$. If the left $K$-module coalgebra $D$ is cosmash (see Theorem 3.1), so is $C$.

Take $K \subset A$ in Theorem 3.1 as $D \subset C$ in Theorem 4.1. Then the former theorem immediately follows from the latter together with the next lemma.

Lemma 4.2. Let $A$ be a Hopf algebra, and $B \subset A$ a Hopf subalgebra including Corad $A$. Then the left $B$-module coalgebra $A$ is cocleft.

Proof. Since the right $B$-module $A$ is free by [T2, Prop. 3], and hence faithfully flat, we see as in the proof of Theorem 3.2 that there is a left $B$-linear and right $A / B^{+} A$ colinear isomorphism $B \otimes A / B^{+} A \cong A$, and so the lemma follows by [MD, Prop. 3.2]; cf. Section 1.

Proof of Theorem 4.1. We will prove that a given $K$-linear coalgebra map $\beta: D \rightarrow K$ can be extended to such a map $C \rightarrow K$.

The wedge [Sw2, Sect. 9.0; Mo, Sect. 5.2]

$$
D \wedge D=\{c \in C \mid \Delta(c) \in D \otimes C+C \otimes D\}
$$

is a left $K$-module coalgebra such that $D \subset D \wedge D \subset C$, which is obviously cocleft. Since the assumption Corad $C \subset D$ implies that $D$ cogenerates $C$, we may suppose $C=D \wedge D$.

Let $\beta: D \rightarrow K$ be as above. There is an invertible $K$-linear map $\gamma: C \rightarrow K$ which preserves the counit. This can be chosen so that $\left.\gamma\right|_{D}=\beta$. To see this, write

$$
E=C / K^{+} C, \quad F=D / K^{+} D \text {. }
$$

Then the natural coalgebra map $F \rightarrow E$ is an injection, since the induced map $K \otimes F \rightarrow$ $K \otimes E$ is identified with the inclusion $D \hookrightarrow C$ through two isomorphisms,

$$
\tilde{\gamma}: C \stackrel{\cong}{\longrightarrow} K \otimes E, \quad \tilde{\gamma}(c)=\gamma\left(c_{1}\right) \otimes \bar{c}_{2}
$$

and an analogous $D \stackrel{\cong}{\longrightarrow} K \otimes F$. For $\beta, \gamma$ as above, the counit-preserving, invertible linear map $\left(\left.\gamma\right|_{D}\right)^{-} * \beta: D \rightarrow K$ annihilates $K^{+} D$, and hence can be extended to such a map $\mu: C \rightarrow K$ annihilating $K^{+} C$; it is indeed invertible since Corad $E \subset F$ (see [Mo, Lemma 5.2.10]). By replacing $\gamma$ with $\gamma * \mu$, we may suppose $\left.\gamma\right|_{D}=\beta$.

The isomorphism $\tilde{\gamma}$ above gives an isomorphism of left $K$-module coalgebras from $C$ to the crossed coproduct $K^{\tau} \ll E$ given by

$$
\begin{aligned}
\rho: E \rightarrow E \otimes K, & \rho(\bar{c})=\bar{c}_{2} \otimes \gamma^{-}\left(c_{1}\right) \gamma\left(c_{3}\right), \\
\tau: E \rightarrow K \otimes K, & \tau(\bar{c})=\gamma^{-}\left(c_{1}\right)_{1} \gamma\left(c_{2}\right) \otimes \gamma^{-}\left(c_{1}\right)_{2} \gamma\left(c_{3}\right),
\end{aligned}
$$

where $c \in C$. Recall these satisfy the three conditions given by [AD, (2.17)-(2.19)].

Notice $\rho$ induces a linear map

$$
\hat{\rho}: E / F \rightarrow E / F \otimes K
$$

Since $\left.\gamma\right|_{D}$ is a coalgebra map, we have $\left.\tau\right|_{F}=\varepsilon(=\varepsilon(\quad) 1 \otimes 1)$. So, $\hat{\tau}:=\tau-\varepsilon$ annihilates $F$, whence it is regarded as a linear map

$$
\hat{\tau}: E / F \rightarrow K \otimes K .
$$


Since $E=F \wedge F$, we see $(\hat{\rho} \otimes \hat{\tau}) \circ \Delta_{E}$ and $(\hat{\tau} \otimes \hat{\rho}) \circ \Delta_{E}$ are both zero, where $\hat{\rho}$ and $\hat{\tau}$ are regarded as linear maps on $E$ annihilating $F$. From the twisted comodule condition $[\mathrm{AD},(2.19)]$ for $\rho$ and $\tau$, it follows that

$$
(\mathrm{id} \otimes \Delta) \circ \hat{\rho}=(\hat{\rho} \otimes \mathrm{id}) \circ \hat{\rho}: E / F \rightarrow E / F \otimes K \otimes K,
$$

whence $(E / F, \hat{\rho})$ forms a right $K$-comodule. We regard it as a $K$-bicomodule with the trivial left $K$-comodule structure. From the normalization condition and the dual cocycle condition $[\mathrm{AD},(2.17),(2.18)]$, it follows that $\hat{\tau}$ is a normalized Hochschild 2-cocycle, by which we mean a 2-cocycle in the normalized standard complex for computing the Hochschild cohomology [D2, Sect. 3.1]

$$
H H^{\cdot}(E / F, K)=\operatorname{Ext}_{K^{e}}(E / F, K)\left(K^{e}=K^{\mathrm{cop}} \otimes K\right) .
$$

By [D2, Thm. 6], $H H^{n}(E / F, K)$ is naturally isomorphic to $\operatorname{Ext}_{K}^{n}(E / F, k)$, which is zero in $n=2$ by assumption. Hence, $\hat{\tau}$ is a coboundary, so there is a linear map $\hat{\nu}: E \rightarrow K$ annihilating $F$ such that $\varepsilon \circ \hat{\nu}=0$ and

$$
\hat{\tau}(x)=-(\hat{\nu} \otimes \mathrm{id}) \circ \hat{\rho}(x)+\Delta \circ \hat{\nu}(x)-1 \otimes \hat{\nu}(x)(=-d \hat{\nu}(x))
$$

for $x \in E$. Define $\nu=\hat{\nu}+\varepsilon: E \rightarrow K$. Then $\varepsilon \circ \nu=\varepsilon$, and $\nu$ is invertible since $\left.\nu\right|_{F}=\varepsilon$.

Define

$$
\gamma^{\prime}=\gamma * \nu: C \rightarrow K
$$

This is a counit-preserving, invertible $K$-linear map such that $\left.\gamma^{\prime}\right|_{D}=\beta$. Let $\rho^{\prime}, \tau^{\prime}, \hat{\rho}^{\prime}, \hat{\tau}^{\prime}$ be the linear maps arising from $\gamma^{\prime}$, as $\rho, \tau, \hat{\rho}, \hat{\tau}$ from $\gamma$. The relations between $(\rho, \tau)$ and $\left(\rho^{\prime}, \tau^{\prime}\right)$ are given by $[\mathrm{AD},(3.1 .4),(3.1 .5)]$, which are dual to [D3, (7), (8)]. They imply that $\hat{\rho}^{\prime}=\hat{\rho}$ and $\hat{\tau}^{\prime}=\hat{\tau}+d \hat{\nu}=0$. Hence, $\tau^{\prime}=\varepsilon$. This proves that $\gamma^{\prime}$ is a coalgebra map, as desired.

REMARK 4.3. In Theorem 4.1, suppose $K$ and $C$ are cocommutative. As is seen from the proof above, the conclusion of the theorem holds true, even if we weaken the assumption $\operatorname{Ext}_{K}^{2}(, k)=0$ so that any symmetric Hochschild 2-cocycle $k \rightarrow K \otimes K$ for the trivial $K$-bicomodule $k$ is a coboundary. This weaker assumption is satisfied, for example, if the coalgebra $K$ is isomorphic to the cofree pointed irreducible cocommutative coalgebra $B(U)$ for some vector space $U$ [Sw2, Sect. 12.2]. Here a Hochschild 2-cocycle is said to be symmetric if it is invariant under composition with the twist map on $K \otimes K$.

5. Vanishing of the cohomology associated to a Singer pair. Let $(H, K, \rightarrow, \rho)$ be a Singer pair [M2, Def. 3.3] of Hopf algebras; it was originally called an abelian matched pair by W. Singer [Si] (see also [T3]). Thus, $H$ is a cocommutative Hopf algebra, $K$ is a commutative Hopf algebra, $\rightarrow: H \otimes K \rightarrow K$ is a module-algebra structure, and $\rho: H \rightarrow H \otimes K$ is a comodule-coalgebra structure, which satisfy together the compatibility conditions given by (1), (2) in [M2, Def. 3.3]. Consequently, $\varepsilon(h \rightarrow x)=\varepsilon(h) \varepsilon(x)$ for $h \in H, x \in K$, and $\rho(1)=1 \otimes 1$ [T3, Lemma 1.2].

Associating to the Singer pair, [M2, p. 3856] constructs, following [Si], a double cosimplicial abelian group, and then the associated, normalized double complex. Further by removing the leftmost vertical complex and the lowest horizontal complex, the following complex is obtained. 


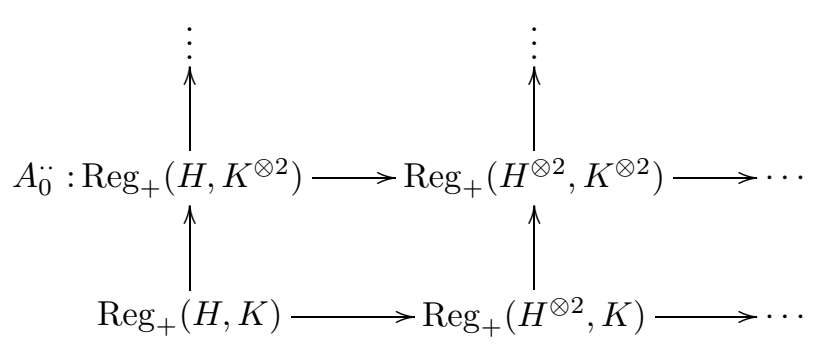

Here, $\operatorname{Reg}_{+}\left(H^{\otimes p}, K^{\otimes q}\right)$ denotes the abelian group of invertible linear maps $H^{\otimes p} \rightarrow K^{\otimes q}$ which are normalized in the sense that they are annihilated by each codegeneracy $s_{i}, t_{j}$ given in [M2, p. 3856]. Let

$$
H^{n}(H, K, \rightarrow, \rho)
$$

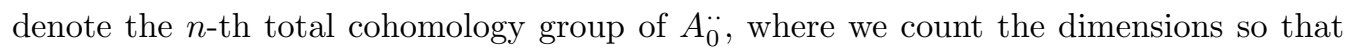
$\bigoplus_{p+q=n+1} \operatorname{Reg}_{+}\left(H^{\otimes p}, K^{\otimes q}\right)$ is the group of total $n$-cochains. Thus, $H^{2}(H, K, \rightarrow, \rho)$ is isomorphic to the group $\operatorname{Opext}(H, K, \rightarrow, \rho)$ of the equivalence classes of those cleft Hopf algebra extensions which are associated to the Singer pair; see [H, Prop. 3.15; M2, Prop. 3.11]. By convention, $H^{0}(H, K, \rightarrow, \rho)=0$.

The lowest horizontal complex

$$
0 \rightarrow k^{\times} \rightarrow \operatorname{Reg}_{+}(H, k) \rightarrow \operatorname{Reg}_{+}\left(H^{\otimes 2}, k\right) \rightarrow \cdots,
$$

which was removed when $A_{0}$ was constructed, is the normalized standard complex [Sw1, p. 210] for computing the Sweedler cohomology $H_{\mathrm{Sw}_{\mathrm{w}}}(H, k)$ with coefficients in the trivial $H$-module algebra $k$. Let

$$
0 \rightarrow k^{\times} \rightarrow \operatorname{Reg}_{+}^{K}(H, k) \rightarrow \operatorname{Reg}_{+}^{K}\left(H^{\otimes 2}, k\right) \rightarrow \cdots
$$

denote the subcomplex of (5.1) which consists of those cochains that are annihilated by the differentials going from (5.1) up to $A_{0}^{.}$. To each $H^{\otimes p}$, a right $K$-comodule (coalgebra) structure is given by $F^{p}(k)$ [M2, p. 3854]; the $K$-coaction on $H^{\otimes p}$ is codiagonal if $\rightarrow$ is trivial. One sees $\operatorname{Reg}_{+}^{K}\left(H^{\otimes p}, k\right)$ consists of all $\varphi \in \operatorname{Reg}_{+}\left(H^{\otimes p}, k\right)$ such that $\varphi\left(x_{0}\right) x_{1}=$ $\varphi(x) 1$ for any $x \in H^{\otimes p}$.

Proposition 5.3. Let $(H, K, \rightarrow, \rho)$ be as above. Suppose $H$ is irreducible and $K$ is cosemisimple. Then $H^{n}(H, K, \rightarrow, \rho)$ is naturally isomorphic to the $n$-th cohomology of the quotient complex of (5.1) by (5.2).

Proof. We will use again the idea used in the proof of Theorem 4.1, 'approximation by the Hochschild cohomology'.

Fix $m>0$. Write $Z=H^{\otimes m}$; this is a right $K$-comodule coalgebra which is pointed irreducible. Let

$$
B_{Z}^{*}: 0 \rightarrow \operatorname{Reg}_{+}(Z, k) \stackrel{\partial}{\longrightarrow} \operatorname{Reg}_{+}(Z, K) \stackrel{\partial}{\longrightarrow} \operatorname{Reg}_{+}\left(Z, K^{\otimes 2}\right) \stackrel{\partial}{\longrightarrow} \cdots
$$

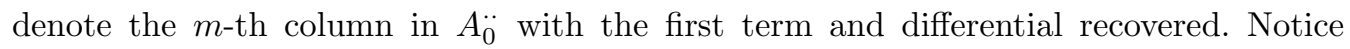
that this is not the normalized standard complex for computing the Doi cohomology $H_{\text {Doi }}(Z, K)\left(=\right.$ Coalg- $\left.H^{\cdot}(Z, K)[\mathrm{D} 1]\right)$, but a subcomplex because every cochain in $B_{Z}$ should be annihilated also by the horizontal codegeneracies $s_{i}$. It suffices to prove $B_{Z}^{\cdot}$ 
is exact everywhere except at $\operatorname{Reg}_{+}(Z, k)$, since then the usual spectral sequence of $A_{0}$ collapses and $E_{2}^{n, 0}$ is the $n$-th cohomology of the quotient complex (5.1)/(5.2).

Let $Z^{\prime}$ denote the sum $\sum_{p=1}^{m} H^{\otimes(p-1)} \otimes k \otimes H^{\otimes(m-p)}$; this is a $K$-costable subcoalgebra of $Z$. Let $D \subset Z$ be a $K$-costable subcoalgebra. Define a complex $B_{D}$ as an analogue of $B_{Z}$ so that $B_{D}^{n}=\operatorname{Reg}_{+}\left(D, K^{\otimes n}\right)$ consists of the normalized standard $n$-cochains for $H_{\text {Doi }}^{\cdot}(D, K)$ that are trivial on $D \cap Z^{\prime}$.

Claim. The restriction map $\operatorname{Reg}_{+}\left(Z, K^{\otimes n}\right) \rightarrow \operatorname{Reg}_{+}\left(D, K^{\otimes n}\right)$ is a surjection.

To prove this, let $\varphi \in \operatorname{Reg}_{+}\left(D, K^{\otimes n}\right)$. Let $\psi: Z \rightarrow K^{\otimes n}$ be a linear map with $\left.\psi\right|_{D}=\varphi$, which is necessarily invertible. We will first construct an extension $\psi_{+}: Z \rightarrow K^{\otimes n}$ of $\varphi$ which is annihilated by each horizontal codegeneracy $s_{i}$ in the double cosimplicial abelian group given in [M2, p. 3856]. For the largest $i$ with $s_{i} \psi \neq \varepsilon$, replace $\psi$ with $\psi^{\prime}:=\psi * d_{i}\left(s_{i} \psi\right)^{-}$, where $d_{i}$ denotes the $i$-th horizontal coface in the abelian group above. Since $\psi=\varepsilon$ on $D \cap Z^{\prime},\left.\psi^{\prime}\right|_{D}=\varphi$. It follows from the relations (see [W, Cor. 8.1.4], for example) among $s_{i}, d_{i}$ that $s_{j} \psi^{\prime}=\varepsilon$ for $j \geq i$. Therefore we obtain desired $\psi_{+}$by repeating this procedure. By the same procedure for the vertical arrows in the abelian group above, we obtain from $\psi_{+}$an extension of $\varphi$ in $\operatorname{Reg}_{+}\left(Z, K^{\otimes n}\right)$. This proves the claim.

Define $C=D \wedge D$; this is a $K$-costable subcoalgebra of $Z$, and so we have a complex $B_{C}$. Fix $n>0$ and a cocycle $\tau$ in $B_{C}^{n}$. Notice that if $D=k^{\otimes m}(=\operatorname{Corad} Z)$, the group $B_{D}^{n}$ is trivial. Therefore, to prove the exactness at $B_{Z}^{n}$, it suffices to see that if $\left.\tau\right|_{D}=\partial \mu$ for some $\mu \in B_{D}^{n-1}$, there exists $\nu \in B_{C}^{n-1}$ such that $\left.\nu\right|_{D}=\mu$ and $\tau=\partial \nu$. Since by the claim above, there is an extension $\mu^{\prime}$ of $\mu$ in $B_{C}^{n-1}$, we can replace $\tau$ with $\tau *\left(\partial \mu^{\prime}\right)^{-}$, and suppose $\mu=\varepsilon$ and $\left.\tau\right|_{D}=\varepsilon$.

Define

$$
E=C / C \cap Z^{\prime}, \quad F=D / D \cap Z^{\prime} .
$$

These are right $K$-comodules with $E \supset F$. We regard the quotient $K$-comodule $E / F$ as a $K$-bicomodule with the trivial left $K$-comodule structure. Regard a linear map $\hat{\varphi}: E / F \rightarrow K^{\otimes n}$ as a linear map $C \rightarrow K^{\otimes n}$ annihilating $D+C \cap Z^{\prime}$, and assign to it $\hat{\varphi}+\varepsilon: C \rightarrow K^{\otimes n}$. We see the assignment gives rise to an isomorphism from the normalized standard complex for computing $H^{\cdot}(E / F, K)$ onto the kernel of the restriction map $B_{C} \rightarrow B_{D}^{*}$; cf. the proof of Theorem 4.1 .

It follows that for $\tau$ as above, there exists a normalized Hochschild $n$-cocycle $\hat{\tau}$ such that $\tau=\hat{\tau}+\varepsilon$. Since $K$ is cosemisimple, there is a normalized Hochschild $(n-1)$-cochain $\hat{\nu}$ such that $\hat{\tau}=d \hat{\nu}$. Then $\nu:=\hat{\nu}+\varepsilon$ in $B_{C}^{n-1}$ has the desired property that $\left.\nu\right|_{D}=\varepsilon$ and $\tau=\partial \nu$.

Corollary 5.4. Let $(H, K, \rightarrow, \rho)$ be a Singer pair, as above. Suppose $H$ is irreducible, $\rho$ is trivial, and $\operatorname{Ext}_{K}^{q}(k, k)=0$ for $0<q \leq n$, where $n>0$ is a fixed integer. Then

$$
H^{q}(H, K, \rightarrow, \rho)=0 \quad(0 \leq q \leq n) .
$$

Proof. Since $\rho$ is trivial, $\operatorname{Reg}_{+}^{K}\left(H^{\otimes p}, k\right)=\operatorname{Reg}_{+}\left(H^{\otimes p}, k\right)$. Hence the corollary follows from (the proof of) the preceding proposition. 
This generalizes [M2, Cor. 4.13] in Case (2). The same result in Case (1) is generalized by the following.

Proposition 5.5. Suppose ch $k=0$. Let $(H, K, \rightarrow, \rho)$ be a Singer pair in which $\rightarrow i s$ trivial. Suppose $H=U(\mathfrak{g})$, the universal envelope of a Lie algebra $\mathfrak{g}$ (possibly of infinite dimension). If the Lie cohomologies $H^{q}(\mathfrak{g}, k)=0$ for $0<q \leq n$, where $n>0$ is a fixed integer, then

$$
H^{q}(H, K, \rightarrow, \rho)=0 \quad(0 \leq q \leq n) .
$$

Proof. By [M2, Prop. 3.14], the double complex $A_{0}^{*}$ is isomorphic to $B_{0}^{*}$ given loc. cit., in which each row is exact up to the $n$-th term by the assumption of Lie cohomologies vanishing. So, the proposition follows in the same way as the preceding corollary.

The final remark below gives a precise information on $k$ for which Sullivan's Theorem (Theorem 3.3) holds true.

RemarK 5.6. Suppose $\operatorname{ch} k=p>0$. Let $H=k[x] /(f(x))$ be the quotient Hopf algebra of the polynomial Hopf algebra $k[x]$ (with $x$ primitive) by the Hopf ideal which is generated by the primitive $f(x)$ given by (3.4). Suppose $K$ is a commutative cosemisimple Hopf algebra containing such a grouplike $g$ that satisfies (3.6). We see $\rho(x)=x \otimes g$ defines a comodule-bialgebra structure $\rho: H \rightarrow H \otimes K$, so that $(H, K, \rightarrow, \rho)$ forms a Singer pair, where $\rightarrow$ is trivial. For $c \in k$, let $A_{c}$ denote the commutative Hopf algebra including $K$ which is generated over $K$ by an element $X$, and defined by (3.5), (3.7); this obviously forms a cleft Hopf algebra extension associated to the Singer pair. One sees $c \mapsto A_{c}$ induces a group homomorphism

$$
\Phi: k \rightarrow \operatorname{Opext}(H, K, \rightarrow, \rho) .
$$

This is a surjection as is seen from the proof of Sullivan's Theorem. We will see that if $g^{q} \neq 1$, the kernel Ker $\Phi$ equals $f(k)=\{f(b) \mid b \in k\}$, and so

$$
\operatorname{Opext}(H, K, \rightarrow, \rho) \cong k / f(k) \text {. }
$$

If $\chi: A_{c} \stackrel{\cong}{\longrightarrow} A_{0}$ gives an equivalence, then $\chi(X)=X+b(1-g)$ for some $b \in k$, and so $c\left(1-g^{q}\right)=\chi(f(X))=f(b)\left(1-g^{q}\right)$. If $g^{q} \neq 1$, this implies $c \in f(k)$, and so Ker $\Phi \subset f(k)$. The converse inclusion always holds true.

Therefore the conclusion of Sullivan's Theorem holds true if and only if $f(k)=k$ for any $f(x)$ of the form (3.4). This is equivalent to that $k$ is algebraically closed, if it is algebraic over the prime field.

Acknowledgments. I thank the referee for his or her kind suggestions, which greatly helped me to improve the manuscript. I also want to thank Piotr Hajac, Wiesław Pusz and S. L. Woronowicz for their warm hospitality in Warsaw.

\section{References}

[A] E. Abe, Hopf Algebras, Cambridge Univ. Press, Cambridge, 1980.

[AD] N. Andruskiewitsch and J. Devoto, Extensions of Hopf algebras, St. Petersburg Math. J. 7 (1996), 17-52. 
[BCM] R. J. Blattner, M. Cohen and S. Montgomery, Crossed products and inner actions of Hopf algebras, Trans. Amer. Math. Soc. 298 (1986), 671-711.

[BM] R. J. Blattner and S. Montgomery, Crossed products and Galois extensions of Hopf algebras, Pacific J. Math. 137 (1989), 37-54.

[C] W. Chin, Crossed products of semisimple cocommutative Hopf algebras, Proc. Amer. Math. Soc. 116 (1992), 321-327.

[DMR] S. Dăscălescu, G. Militaru and S. Raianu, Crossed coproducts and cleft coextensions, Comm. Algebra 24 (1996), 1229-1243.

[DG] M. Demazure and P. Gabriel, Groupes Algébriques I, North-Holland, Amsterdam, 1970.

[D1] Y. Doi, Cohomologies over commutative Hopf algebras (with appendix by M. Takeuchi), J. Math. Soc. Japan 25 (1973), 680-706.

[D2] Y.Doi, Homological coalgebra, J. Math. Soc. Japan 33 (1981), 31-50.

[D3] Y.Doi, Equivalent crossed products for a Hopf algebra, Comm. Algebra 17 (1989), 3053-3085.

[DT] Y.Doi and M. Takeuchi, Cleft comodule algebras for a bialgebra, Comm. Algebra 14 (1986), 801-818.

[H] I. Hofstetter, Extensions of Hopf algebras and their cohomological description, J. Algebra 164 (1994), 264-298.

[KT] H.F.Kreimer and M. Takeuchi, Hopf algebras and Galois extensions of an algebra, Indiana Univ. Math. J. 30 (1981), 675-692.

[Mj1] S. Majid, More examples of bicrossproducts and double cross product Hopf algebras, Israel J. Math. 72 (1990), 133-148.

[Mj2] S. Majid, Foundations of Quantum Group Theory, Cambridge Univ. Press, Cambridge, 1995.

[M1] A. Masuoka, Quotient theory of Hopf algebras, in: J. Bergen and S. Montgomery (eds.), Advances in Hopf Algebras, Lec. Notes in Pure and Appl. Math. 158, Marcel Dekker, New York, 1994, 107-133.

[M2] A. Masuoka, Extensions of Hopf algebras and Lie bialgebras, Trans. Amer. Math. Soc. 352 (2000), 3837-3879.

[MD] A. Masuoka and Y. Doi, Generalization of cleft comodule algebras, Comm. Algebra 20 (1992), 3703-3721.

[Mo] S. Montgomery, Hopf Algebras and Their Actions on Rings, CBMS Regional Conf. Ser. in Math. 82, Amer. Math. Soc., Providence, 1993.

[N] M. Nagata, Complete reducibility of rational representations of a matric group, J. Math. Kyoto Univ. 1 (1961), 87-99.

[P] P. Schauenburg, A generalization of Hopf crossed products, Comm. Algebra 27 (1999), 4779-4801.

[S1] H.-J.Schneider, Zerlegbare Erweiterungen affiner Gruppen, J. Algebra 66 (1980), 569593.

[S2] H. -J. Schneider, Normal basis and transitivity of crossed products for Hopf algebras, J. Algebra 152 (1992), 289-312.

[Si] W. Singer, Extension theory for connected Hopf algebras, J. Algebra 21 (1972), 1-16.

[SV] D. Stefan and F. Van Oystaeyen, The Wedderburn-Malcev theorem for comodule algebras, Comm. Algebra 27 (1999), 3569-3581.

[Su] J.Sullivan, A decomposition theorem for pro-affine solvable algebraic groups over algebraically closed fields, Amer. J. Math. 95 (1973), 221-228. 
[Sw1] M. Sweedler, Cohomology of algebras over Hopf algebras, Trans. Amer. Math. Soc. 127 (1968), 205-239.

[Sw2] M. Sweedler, Hopf Algebras, Benjamin, New York, 1969.

[Sw3] M. Sweedler, Connected fully reducible affine group schemes in positive characteristic are abelian, J. Math. Kyoto Univ. 11 (1971), 51-70.

[T1] M. Takeuchi, On a semi-direct product decomposition of affine groups over a field of characteristic 0, Tôhoku Math. J. 24 (1972), 453-456.

[T2] M. Takeuchi, Relative Hopf modules-equivalences and freeness criteria, J. Algebra 60 (1979), 452-471.

[T3] M. Takeuchi, Matched pairs of groups and bismash products of Hopf algebras, Comm. Algebra 9 (1981), 841-882.

[W] C. Weibel, Introduction to Homological Algebra, Cambridge Univ. Press, Cambridge, 1994. 